\title{
Uncommon Location of Common Parasite
}

\author{
Sherwani $\mathbf{P}^{1}$, Tomer $\mathbf{S}^{1}$, Pahuja $\mathbf{L}^{2}$, Anand $\mathbf{R}^{1}$ \\ ${ }^{1}$ Lady Hardinge Medical College and Associated Hospitals, New Delhi, ${ }^{2}$ Department of Radio \\ diagnosis, Chacha Nehru Bal Chikitsalya Hospital, Delhi, India
}

\begin{abstract}
Neurocysticercosis is the most common parasitic infection of the Central Nervous System caused by Taenia solium. Spinal cysticercosis is an uncommon site of cysticercal infestation $\&$ intramedullary involvement is even rarer. ${ }^{1} \mathrm{We}$ are reporting a rare case of intramedullary cysticercosis in the cervical region in an 11 year old girl who presented with headache \& neck stiffness. CT and MRI of the cervical spine done revealed a cystic lesion with mural nodule (scolex) with cord edema in the cervical region. The child underwent treatment with antihelminthic drugs and showed marked clinico-radiological improvement.
\end{abstract}

Keywords: Intramedullary, Neurocysticercosis (NCC), Spinal Cord.

\section{Introduction}

Neurocysticercosis, causative agent, Taenia solium, is the most common parasitic infection affecting the central nervous system. Spinal cysticercosis is rare, which accounts for only $1.2 \%$ to $5.8 \%$ of all cases of neurocysticercosis. ${ }^{2,3}$

Spinal cysticercosis can be classified according to its location as extraspinal (vertebral) or intraspinal which can be further classified into epidural, subdural, arachnoid or intramedullary. Out of which intramedullary type is very rare. ${ }^{4,5,6}$ Here we report a rare case of intramedullary

Corrospondence to: Dr Poonam Sherwani, Department of Radiodiagnosis, Lady Hardinge Medical College and associated Hospitals, New Delhi-110001.

Email Id: Poonam_doc2003@yahoo.co.in cysticercosis in a 11 year old child in cervical region and discuss its imaging and literature.

\section{Case Report}

A 11 year old girl was referred to our hospital with complaints of severe headache, giddiness, vomiting and neck stiffness since 1 week along with weakness in bilateral upper limbs. The headache was localized to fronto-parietal and occipital region and was throbbing in nature. On examination, her vitals were stable. On neurological examination, the child had neck stiffness and Kernig's sign was present. There were no clinical signs of cranial nerve palsy. The patient had power $4 / 5$ in bilateral upper limbs. On examination, her vitals were stable. Haemogram was within normal limits. CSF sugar and protein was normal. The child was further evaluated in our 

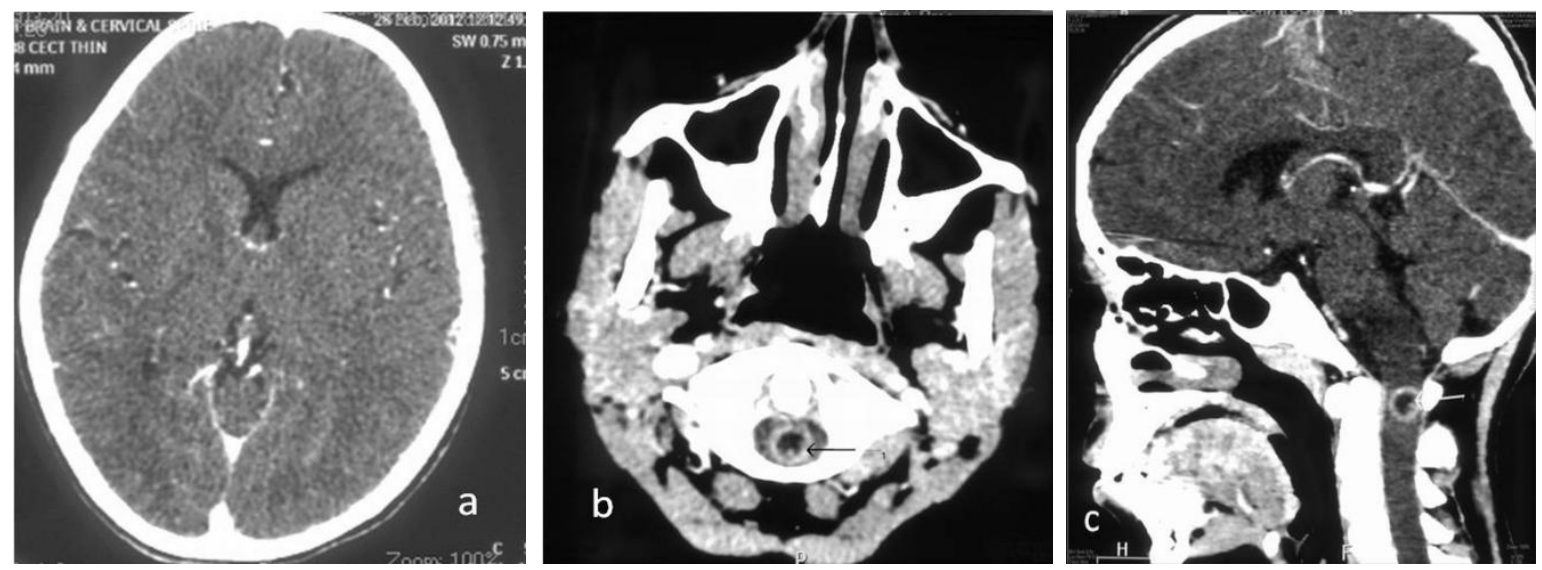

Fig. 1: CECT Brain shows normal brain parenchyma and ventricular system with no intraparenchymal focal lesion.(a) Contrast enhanced CT axial (b) and sagittal (c) reformatted images of cervical spine in soft tissue window shows intraspinal peripheral enhancing hypodense lesion (arrow in b) at the level of $\mathrm{C1}$ vertebra with hyperdense eccentric nodule.(arrow in c).

department for various radiological investigations. X-Ray cervical spine and chest X-Ray PA was done which did not reveal any significant abnormality. The child was further evaluated with CT scan. NCCT followed by CECT brain and cervical spine was done on multislice CT Philips Brilliance 16 in our department. CT brain study did not reveal any focal parenchymal lesion or any abnormal meningeal enhancement (Fig.1a).

NCCT followed by contrast enhanced cervical spine ( soft tissue window) revealed $9.6 \times 9.8 \mathrm{~mm}$ sized ring enhancing lesion in the spinal canal at $\mathrm{C} 1$ vertebral level with suspicious hyperdense eccentric mural nodule in it (Fig. 1b \& Fig1c).

No evidence of any underlying bone abnormality was seen. Based on CT imaging, the diagnosis of infective granuloma was given. For further evaluation, MRI Cervical spine was done. T1W, T2W and GRE sequence was done in different planes. T2W axial and sagittal sections showed intramedullary focal lesion at $\mathrm{C} 1$ vertebral level with hyperintense core and peripheral

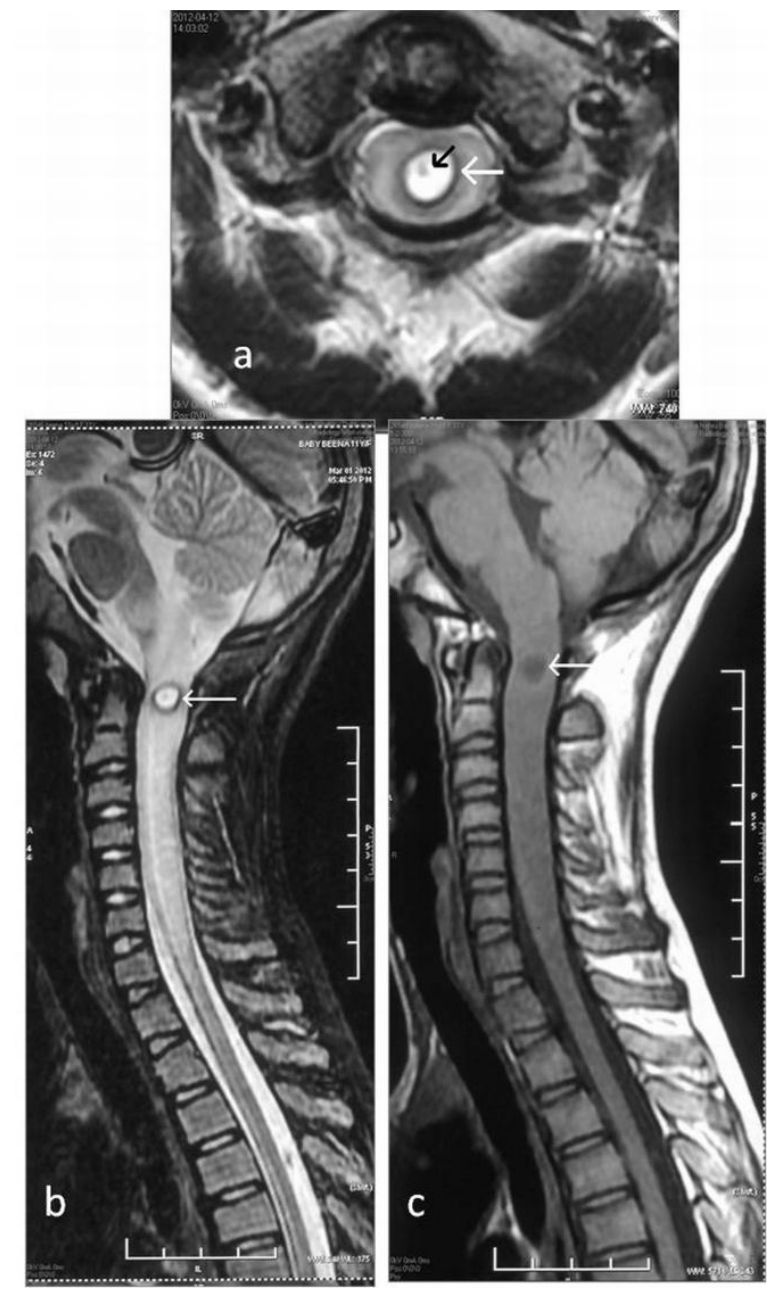

Fig. 2: T2W axial (a) and Sagittal (b) MR image shows well defined rounded intramedullary focal lesion (white arrow) with surrounding hypointense rim and 
hyperintense core with a hypointense eccentric mural nodule (black arrow) at C1 level. Diffuse cord edema is seen extending from the level of medulla to D1 veretebra. The lesion (arrow) appears hypointense on T1(c).

hypointense rim with eccentric hypointense nodule in it. Surrounding cord edema is seen extending from the level of medulla to D1 vertebral level (Fig. 2a \& Fig.2b). The lesion appeared hypointense on T1W images (Fig. 2c).

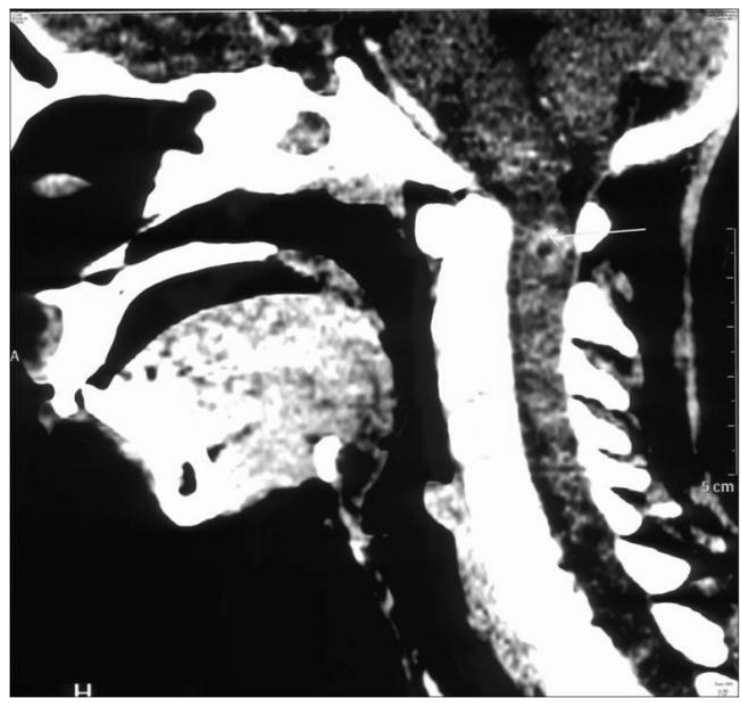

Fig. 3: Follow up CECT neck in reformatted sagittal image which shows there is regression in the size of the lesion (arrow). The lesion is degenerating and walls of the lesion have become irregular. Scolex is no longer seen.

A diagnosis of intramedullary cysticercosis was made. The child was put on antihelminthic drugs and prednisolone for 4 weeks and the child showed clinicoradiological improvement.As patient could not afford MRI, follow up NCCT scan of the cervical spine after 4 weeks was done which showed regression in the size of the lesion 7x7mm (Fig. 3).

\section{Conclusion}

Intramedullary cysticercosis is an extremely rare entity with an incidence reported between $0.7 \%$ and $5.86 \%$. Intramedullary cysticercosis often presents in patients between 20 to 45 years old and is very rare in children. ${ }^{7}$ Most of the patients present with pain, paraparesis, spasticity, and bowel and bladder incontinence. Four forms of spinal neurocysticercosis can be seen - vertebral, extradural and intradural extramedullary and intramedullary. The commonest is intradural extramedullary type. ${ }^{1}$ Half of the cases have concomitant extraspinal involvement.

Extramedullary forms occur due to dissemination through CSF through the focus in brain, whereas intramedullary form occurs due to hematogenous spread of the parasitic laravae. The thoracic cord is the most common site for intramedullary cysticercosis due to its high vascularity and for the same reason, involvement of brain is commoner than the spinal one. ${ }^{3}$

CSF examination in patient with NCC reveals moderately elevated lymphocytes, eosinophils, protein with low/ normal glucose levels. CSF ELISA can be done further for confirmation.

MRI is the investigation of choice to diagnose NCC. Usual MR findings are cystic lesion with signal intensity similar to CSF on T1W \& T2W images. Mural nodule (scolex) appears isointense to hyperintense on T1WI and hypointense on T2WI as seen in our case. On contrast enhanced MR, the cyst usually shows ring enhancement. Surrounding cord edema is usually seen. Our case also depicted the similar MRI findings. MRI helps not only in the diagnosis but also 
in the preoperative localization of the lesion and even the extent of involvement. The definitive diagnosis is presence of scolex and if the scolex is not seen, the differentials include Tubercular granuloma, Hydatid cyst, Dermoid cyst, Syrinx and metastasis.

Surgery can relieve the compressive element in the patients presenting with acute onset of symptoms and in cases where diagnosis is not certain. ${ }^{8}$ However operative mortality is around $15 \%$ and morbidity is reported to be $8.5 \%{ }^{3} \quad$ Medical treatment include antihelminthic drugs along with steroids which reduces inflammation and also increases the blood levels of albendazole and duration varies from 4-8 weeks. Our patient was treated with albendazole and steroids and showed marked clinicoradiological improvement.

To conclude, isolated intramedullary cysticercosis is very rare, however correlation of clinical findings along with imaging helps in making the diagnosis and the appropriate treatment. Our case is unique as presentation in paediatric age group is rare as well as the site of involvement (cervical spine) which is seen in our case is even rarer in cases of spinal cysticercosis.

\section{References}

1. Shah FA, Kirmani S, Badar F, Ahmed I. Isolated intramedullary spinal cysticercosis in a 10 year old female showing dramatic response with albendazole. J Paediatr Neurosci. 2011; 6(1):52-54.

2. Sawhney IM, Singh G, Lekhra OP. et al. Uncommon presentation of neurocysticercosis. J Neurol Sci. 1998;154(1):94-100.
3. Sharma BS, Banerjee AK, Kak VK. Intramedullary spinal cysticercosis: case report and review of literature. Clin Neurol Neurosurg. 1987;89(2):111-6.

4. Agrawal R, Chauhan SP, Misra V. et al. Focal spinal intramedullary cysticercosis. Acta Biomed. 2008;79(1):39-41.

5. Ahmad FU, Sharma BS. Treatment of intramedullary spinal cysticercosis: report of 2 cases and review of literature. Surg Neurol. 2007;67(1):74-7.

6. Chhiber SS, Singh B, Bansal P. et al. Intramedullary spinal cysticercosis cured with medical therapy: case report and review of literature. Surgical Neurology. 2009;72(6):765-9.

7. Mathuriya SN, Khosla VK, Vasishta RK. et al. Intramedullary cysticercosis: MRI diagnosis. Neurol India. 2001;49(1):71-4.

8. Holtzman RN, Hughes JE, Sachdev RK, Jarenwattananon A. An intramedullary cysticercosis. Surg Neurol. 1986;26:18791. 